\title{
Planning and Estimation of Operations Support Requirements
}

\author{
Marilyn E. Newhouse* \\ CSC \\ Marshall Space Flight Center, Alabama, 35812, USA \\ Bryan Barley $^{\dagger}$, Allen Bacskay ${ }^{\ddagger}$, Dennon Clardy ${ }^{\S}$ \\ National Aeronautics and Space Administration, George C. Marshall Space Flight Center, \\ Marshall Space Flight Center, Alabama, 35812, USA
}

\begin{abstract}
Life Cycle Cost (LCC) estimates during the proposal and early design phases, as well as project replans during the development phase, are heavily focused on hardware development schedules and costs. Operations (phase E) costs are typically small compared to the spacecraft development and test costs. This, combined with the long lead time for realizing operations costs, can lead to de-emphasizing estimation of operations support requirements during proposal, early design, and replan cost exercises. The Discovery and New Frontiers (D\&NF) programs comprise small, cost-capped missions supporting scientific exploration of the solar system. Any LCC growth can directly impact the programs' ability to fund new missions, and even moderate yearly underestimates of the operations costs can present significant LCC impacts for deep space missions with long operational durations. The National Aeronautics and Space Administration (NASA) D\&NF Program Office at Marshall Space Flight Center (MSFC) recently studied cost overruns and schedule delays for 5 missions. The goal was to identify the underlying causes for the overruns and delays, and to develop practical mitigations to assist the D\&NF projects in identifying potential risks and controlling the associated impacts to proposed mission costs and schedules. The study found that 4 out of the 5 missions studied had significant overruns at or after launch due to underestimation of the complexity and supporting requirements for operations activities; the fifth mission had not launched at the time of the mission. The drivers behind these overruns include overly optimistic assumptions regarding the savings resulting from the use of heritage technology, late development of operations requirements, inadequate planning for sustaining engineering and the special requirements of long duration missions (e.g., knowledge retention and hardware/software refresh), and delayed completion of ground system development work. This paper updates the D\&NF LCC study, looking at the operations (phase E) cost drivers in more detail and extending the study to include 2 additional missions and identifies areas for increased emphasis by project management in order to improve the fidelity of operations estimates.
\end{abstract}

\section{Introduction}

The Discovery Program (DP) is a science program of frequent, mid-class spacecraft missions that perform highquality focused scientific investigations. Initiated in 1992, the DP was defined to ensure frequent access to space for planetary system(s) science investigations, emphasizing missions that can be accomplished under the leadership of the scientific research community. The DP comprises a long-term series of space science missions that are independent and uncoupled, but share a common funding and management structure. Since its inception, DP has successfully completed missions to study the Moon, inner planets, asteroids, comets, and solar wind. Current

\footnotetext{
${ }^{*}$ Principal Lead Systems Engineer; MSFC/VP23.

${ }^{\dagger}$ Lunar Quest Program Chief Engineer, MSFC/EE04.

${ }^{\ddagger}$ Discovery and New Frontiers Deputy Program Manager, MSFC/VP23.

${ }^{\S}$ Discovery and New Frontiers Program Manager, MSFC/VP23.
} 
missions in development or operations will continue exploration of the inner and outer planets, asteroids, comets, and the Moon.

The New Frontiers Program (NFP) is a science program of medium-size spacecraft missions that perform highquality focused scientific investigations. Initiated in 2003, the NFP was defined to pursue planetary missions that require resources beyond those available in the DP. The NFP comprises a long-term series of space science missions that are independent and uncoupled, but share a common funding and management structure. The NFP currently includes two missions to study outer and dwarf planets.

Missions for both programs are selected through an open science competition and can include any science investigation involving solar system objects except for the Earth and the Sun, which are currently covered by other programs. D\&NF missions are Principal Investigator (PI)-led, complete, self-standing, and uncoupled Science Mission Directorate (SMD) investigations. The total cost to the National Aeronautics and Space Administration (NASA) for each full mission has a not-to-exceed cost cap specified in the Announcement of Opportunity (AO) for the competition. At the time of this study, the Discovery mission cost cap was $\$ 425 \mathrm{M}$ and the New Frontiers mission cost cap was $\$ 700 \mathrm{M}$. The mission cost cap covers the complete mission, including spacecraft development, mission operations, data analysis, and education and public outreach.

D\&NF missions are ultimately defined in terms of the science return from the mission. Level I requirements include the baseline science mission: the full set of scientific requirements identified for the mission, and the threshold science mission: the minimum set of science requirements below which the mission is not considered justifiable for the proposed cost. Each PI is held responsible for proper execution of all aspects of the mission, including implementation and execution within the confirmed mission cost and schedule.

The D\&NF programs are managed by a single program office at Marshall Space Flight Center. As uncoupled, multi-mission programs emphasizing cost-capped PI-led missions, the ability of the D\&NF programs to meet their launch frequency requirements is driven by the ability of each individual project to meet its proposed and confirmed LCC and schedule. Looking at the missions over the history of the D\&NF programs showed an increased frequency of cost overruns (see Figure 1).

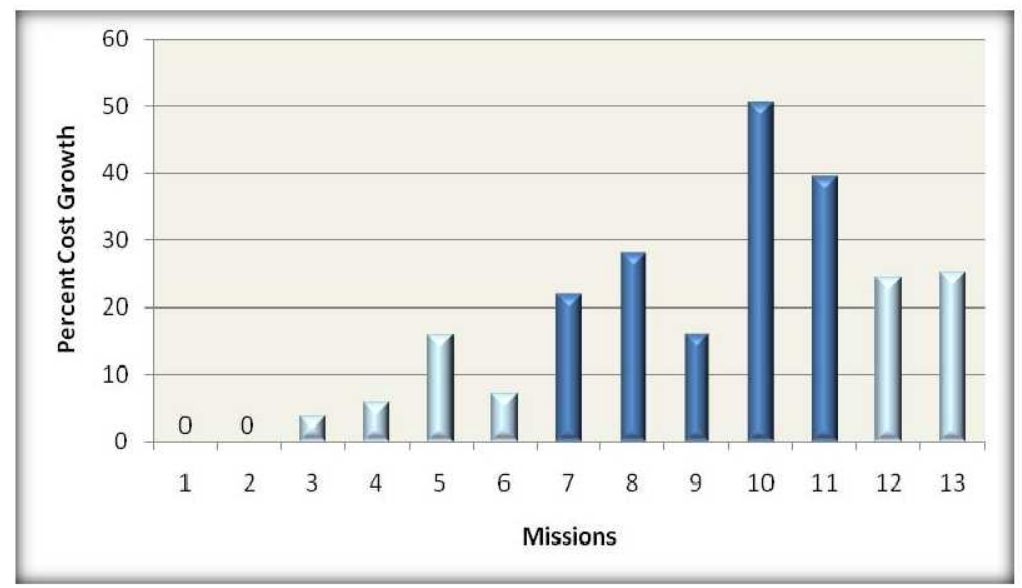

Figure 1. D\&NF Life Cycle Cost Growth

While there was a trend of increasing life cycle cost growth even in the earliest D\&NF missions, the growth was contained in the early missions, showing an average cost growth of $\sim 2 \%$ and a maximum of $15 \%$ for one mission. In contrast, the five recent missions selected for the study showed an average cost growth of $31 \%$, with one mission reaching $50 \%$. This growth directly affects the programs' ability to fund new mission starts and meet their objectives to provide opportunities for the science community to propose and execute solar system exploration missions.

Therefore, the D\&NF Programs Manager looked at the cost escapes on recent D\&NF missions to identify the primary drivers and determine what reasonable things could be done as a program to either prevent the cost escapes or manage them better. Five missions were selected from the two programs based on a recent history of exceeding proposed or confirmed costs. Those missions covered a spectrum of complexity, cost growth, and maturity and are highlighted in dark blue in Figure 1. The study process and results are detailed in the final report, "Improving the Life Cycle Cost Management of Planetary Missions."

The study found that four of the five missions experienced growth in operations costs at or after launch. The study identified the following issues as the primary operations cost growth drivers:

1) Underestimating the complexity of mission operations

2) Inadequate planning for sustaining engineering

3) Ignoring special requirements for long duration missions, e.g., knowledge retention, software and hardware refresh, technology evolution, institutional staffing considerations

The study also indicated that the cost growth could be traced to problems that were embedded as a result of decisions that were made during formulation (phases A and B). In addition, these same drivers were identified as 
issues during project replans, compounded by a separate finding that, in general, insufficient time was allowed during replans to fully develop and analyze impacts to the operations concept resulting from the project replan.

At the time of the D\&NF LCC study, the five missions studied were well into or past the detailed design phase (phase C). Two missions were in operations and one had completed its primary mission. Since that time, the final two missions have launched and are in operations (phase E) and two additional missions have completed their detailed designs (phase C). This paper expands the original study and provides a more detailed look specifically at the operations cost growth drivers experienced by seven recent D\&NF missions, all of which have shown or are showing some growth in operations cost, or a descope of capabilities to contain operations cost growth.

\section{Operations (Phase E) Cost Growth}

The total cost to the National Aeronautics and Space Administration (NASA) for each full D\&NF mission has a not-to-exceed cost cap specified in the AO for the competition, that covers the complete mission, including spacecraft development, mission operations, data analysis, and education and public outreach. At the time of this study, the Discovery mission cost cap was $\$ 425 \mathrm{M}$ and the New Frontiers mission cost cap was $\$ 700 \mathrm{M}$. Operations (phase E) costs for each mission are highly variable depending on the type of mission (flyby, orbiter, sample return, or lander) and the duration of the operational mission. For the missions studied, operations (phase E) costs range from $\$ 18 \mathrm{M}$ to $\$ 174 \mathrm{M}$.

Figure 2 illustrates the growth in Phase E costs for four out of the seven D\&NF missions studied. The primary axis is the phase E status (percent complete); the secondary axis is the percent growth in the operations (phase E) costs. One of the missions has completed its primary science mission (phase $\mathrm{E}$ duration $100 \%$ complete, shown in gray), and therefore will not show any additional cost growth. However, the remaining three missions are still in operations and could experience additional cost growth prior to completion.

Of the missions studied, none showed any significant growth in the operations cost estimates during formulation or design (phases A through C). However, all missions in operations experienced operations cost growth at or after launch. For the one mission in the study that has

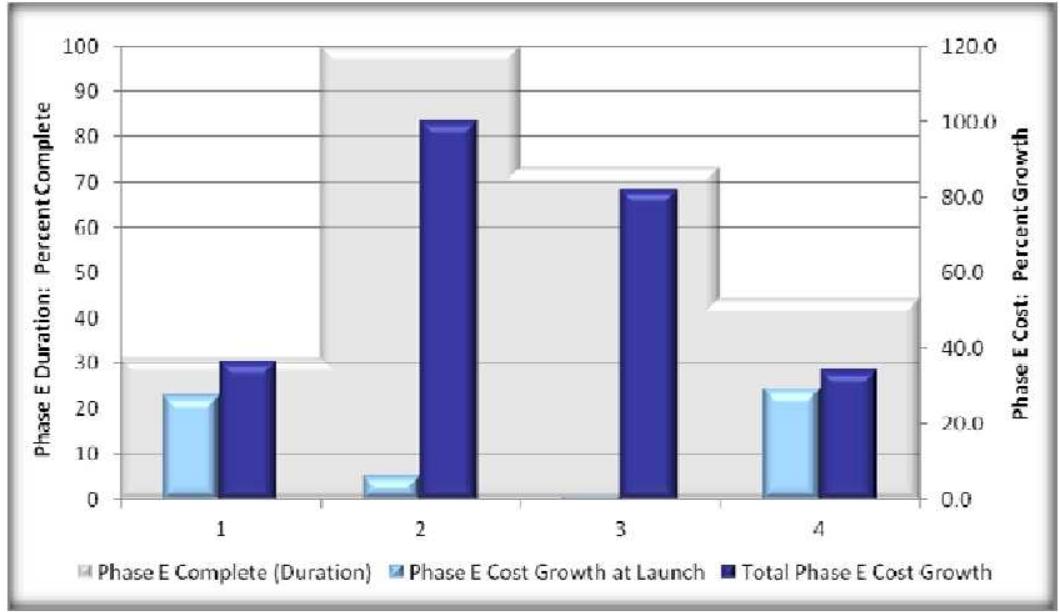

Figure 2. Operations (Phase E) Cost Growth completed its primary science mission, the total operations cost increased $100 \%$ over the original phase E estimate in the proposal.

Of the 3 missions not shown in Figure 2, one has shown little growth in operations cost since launch, but only as a result of a reduction in the science mission duration and a careful replan of residual ground system development work. The remaining two missions have not yet launched and, consistent with the study data shown, neither mission showed any operations cost growth at the end of phase C (Critical Design Review (CDR)). However, one is already showing an increase in the estimate for operations support costs that will be realized by launch.

\section{Operations Drivers}

D\&NF missions share many similar operational characteristics that differ from other types of missions. First, as planetary missions, phase $\mathrm{E}$ is often dominated by a long cruise to the destination target followed by a relatively short period of critical science operations. Second, there is often one and only chance to execute the required science activities. For example, if the science goal is a fly-by of a solar system object, all science data must be collected within a window of hours to weeks, depending on the type of science measurement. There is no opportunity to repeat an observation on a subsequent orbit. Even for missions designed to orbit another solar system object, constraints on mission duration combined with minimum observation durations often result in minimal or no opportunities to revisit or repeat an observation. Third, as the mission progresses, the round-trip time delay for 
command and telemetry increases. Typically, by the start of science operations, the round-trip time delay precludes real-time ground intervention in critical activities (e.g., encounter or orbit insertion).

These characteristics define the ground system tools, processes, and staffing required for D\&NF missions. Some activities and the associated ground system tools (e.g., monitoring spacecraft health and safety) are identical during cruise and science operations, while others (e.g., scheduling) are minimal during cruise and extremely intensive during science operations. Ground system requirements need to take into account the tools and their use in the science phase, as well as the tools required for routine cruise operations. From a process perspective, command loads for critical operations are typically built well ahead of critical spacecraft and science events and thoroughly tested using ground simulators, and then execute onboard without ground intervention. For most critical mission activities (e.g., encounter or deep space maneuvers), the scheduling and load validation can be completed ahead of time and then executed onboard. However, for missions orbiting a solar system object, the need for sustained operational scheduling and load validation levies additional requirements on science phase tools and staffing. Finally, containing operations costs typically dictates minimizing staffing during cruise, but the intensity of operations at the science target dictates a full complement of fully-trained operations personnel able to "hit the ground running."

\section{Cost Growth Drivers}

Cost overruns for the D\&NF missions studied range from $35 \%$ to $100 \%$ of the initial (proposed) operations cost (Figure 2). This study found that the drivers behind these overruns include late identification and underestimation of operations requirements, particularly science requirements and fault management support; underestimation of the overall mission complexity; overly optimistic assumptions about heritage and new technology implementation; development delayed into phase E; development discipline during phase E, overly optimistic assumptions and underestimation of sustaining engineering support and knowledge retention for long duration missions. Figure $\mathbf{3}$ shows how these drivers breakdown across the missions studied; the following paragraphs discuss each of these drivers in more detail. The drivers were apparent both in the initial estimates developed as part of the proposal and concept study development processes, and in updated estimates during project replans. .

\section{A. Estimation}

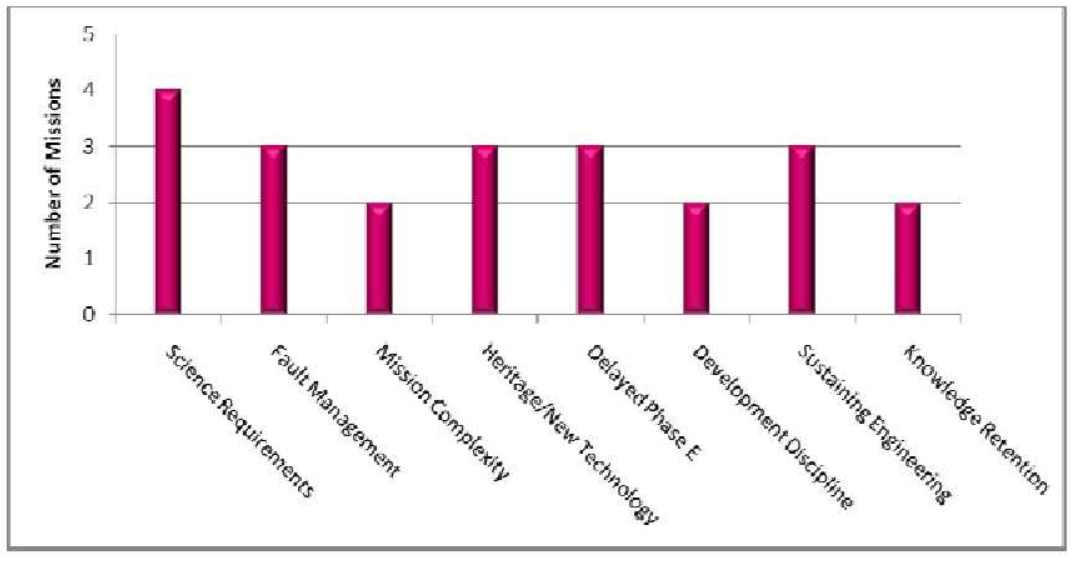

Figure 3. Cost Growth Drivers Identified per Mission

In the missions studied, all five operational missions experienced phase $\mathrm{E}$ cost growth resulting from an underestimate of the operational requirements. The most common area for growth was in the tools required for science operations at the science targets. As solar system exploration missions, the differing science objectives translate into unique science operations activities. This means that while multi-mission operations center capabilities are typically sufficient, with minimal modifications, for routine command and monitoring activities, each mission requires unique tools for science scheduling and operations. D\&NF projects estimated ground system development costs based on reuse of a multi-mission operations center without sufficient detailed requirements and design analysis of the science operations phase. For example, until recently, most small deep space missions have been encounter missions with a long cruise phase for planning and testing command sequences that then execute autonomously during the encounter. Recent missions that include orbital operations for a significant period of time around the science target, require a more extensive suite of tools to support iterative operations more typical for a low earth orbiter than a deep space mission. The growth in requirements when these projects began detailed science planning resulted in significant growth in ground system requirements and development costs, either late in phase D or well into phase $\mathrm{E}$.

The other area that was found to be routinely underestimated was fault protection and fault management. The drivers for growth in this area are discussed in a white paper $^{2}$ summarizing the results of a fault management 
workshop sponsored by NASA's Planetary Science Division in April of 2008. The impact of underestimating fault management capabilities on operations is two-fold. First is the obvious increase to phase E costs to complete development and testing of residual fault management capabilities that are not considered launch critical. However, the hidden cost is the burden on the operations team to execute the workarounds required to ensure the health and safety of the spacecraft while the development of automated capabilities is completed, as well as support for fault management testing prior to operational use. Two of the D\&NF missions required augmented staffing, in one case for up to a year after launch, to support ongoing operations in parallel with testing and validation of fault management capabilities.

D\&NF missions also underestimated the overall complexity of operations activities and therefore the level of support for planning and executing onboard activities. While this was an issue for the initial cost estimates in a mission proposal, for the missions studied it had the largest effect during project replans. For deep space missions, a small change to the launch window can have a significant effect on the mission design (spacecraft trajectory to the science target). In one mission studied, a 3 month launch slip increased the total mission duration by 2 years, the number of critical events by $50 \%$, and introduced a one year period with 4 critical events. However, there was insufficient time during the replan for the project to fully analyze the impacts on operations. After launch, phase $\mathrm{E}$ costs increased $80 \%$ in large part to accommodate the heavier operational load.

In addition to underestimating the effort required to develop new and unique operational capabilities, the D\&NF missions studied experienced cost overruns resulting from overly optimistic assumptions regarding the use of heritage technology without sufficient analysis to confirm the operational characteristics of the technology in the proposed mission environment. D\&NF missions often cite previous mission experience and the resulting lessons learned as justification for streamlined operations during cruise, allowing for reduced staffing over previous missions. However, two missions failed to realize this reduction due to significant changes in thermal or radiation environment from the previous missions. Both missions experienced cost growth for augmented engineering staff to support unique operational activities necessary to ensure the safety of the spacecraft and science instruments or additional analysis to understand the causes and ramifications of mission anomalies.

For another mission, the full characteristics of a relatively new propulsion technology used to enable the science mission were not taken into account during prelaunch planning. During cruise, it became clear that differences in timing between the new and traditional propulsion systems would result in a decrease in usable science observation time at the target and increased operational overhead. The operational overhead and extension of the science mission required increases to the both the science and engineering staff during the approach to and at the science target and an accompanying growth in operations costs.

Projects can also demonstrate overreliance on heritage/multi-mission operations center systems and capabilities. Many of the day-to-day activities required to operate a spacecraft are routine and similar from one spacecraft to the next. For example, the procedures for building and sending commands to a spacecraft, receiving and processing raw spacecraft telemetry, and monitoring spacecraft performance are very similar, if not identical from spacecraft to spacecraft. However, that very similarity can lead to complacency in reusing operations concepts without sufficient analysis to identify key differences, perhaps in timing or complexity, that drive operations support requirements for a specific mission or mission phase. As one example, most spacecraft today are operated from multi-mission operations centers. However, even though the software and hardware in a multi-mission center can be configured easily to operate two different spacecraft, operating two spacecraft in tandem is inherently different from operating two independent spacecraft. The operations concept needs to clearly identify these differences, not just rely on the similarities, in order to drive out resource requirements: software and hardware requirements, configuration or reconfiguration issues, and personnel for critical operations and contingency response.

\section{B. Scheduling}

It is not unusual for deep space missions to plan from the initial concept to take advantage of the long cruise phase to complete ground system capabilities, particularly science phase capabilities. The critical mission operations capabilities (e.g., command and monitoring) are completed prior to launch, but science scheduling and processing capabilities will be deliberately delayed until after launch. While counterintuitive for those who are used to developing ground operations capabilities for low earth orbiting missions, this delayed development has an advantage for deep space mission: the delayed development provides a means for justifying the retention of staff during the long cruise interval, serving to maintain critical knowledge and skills during the cruise to the science target. However, the approach is not without risk.

Given the long delay before implementation of the science tools, there is a significant temptation to underemphasize the science phase requirements and design during project formulation. This can be the result of decisions to limit ground system or science team staffing to control development costs; it can result from the 
pressure of other more time critical spacecraft or ground system issues; or it result from a desire to benefit from the knowledge gained about instrument operations during development, integration, and test. However, letting the science phase requirements and early design work lag behind other spacecraft and ground system, significantly increases the risk that the tools and development effort will be significantly underscoped in early cost estimates, leading to cost growth in phase $\mathrm{E}$.

Also, delaying the development of ground system tools into phase E can result in a decrease in development discipline. Projects in phase $\mathrm{E}$ are rarely required to implement earned value management, a valuable tool for scheduling and tracking development progress. Given the long lead time before tool development, tool designs at the project CDR may not be at the same level of maturity as other areas of the ground system, yet rarely are equivalent reviews, including independent experts, held during operations for the remaining development work. Taken together, the decreased project management visibility into requirements changes and scope increase, along with the lack of design maturing, resulted in tool development cost growth during phase $\mathrm{E}$.

\section{Sustaining Engineering}

Sustaining engineering support is another area that is routinely underestimated for the D\&NF missions studied. Projects do not adequately address requirements for hardware and software refresh over the life of a deep space mission. The primary operational phase of D\&NF missions can last up to 10 years with the majority of the time spent in cruise to the final destination. Yet most missions assume they will use the same ground system hardware (both for operations and simulation/load verification) for the full mission. On the surface, this decision keeps phase $\mathrm{E}$ costs down for both the hardware, software tool changes to adapt to the new configuration, and staffing. It also eliminates the potential mission risk of errors resulting from supporting changes to ground system tools. However, this assumption can become untenable as a result of operating system upgrades and hardware obsolescence. Long term, the assumption can actually increase the risk of loss of science in the event that aging hardware fails during a critical mission event. If a mission is forced into an unplanned upgrade to its hardware/software configuration, the cost impact goes well beyond the specific hardware or operating system costs. The project must then take into account maintenance of the software tools and the increased staffing required to support simultaneous development/testing and operations.

Knowledge retention across the life of a deep space mission is the other area that is routinely underestimated in D\&NF missions. The desire to control costs for routine operations during cruise competes with the need to retain key personnel, skills, and knowledge to meet the demands during critical operations. D\&NF mission proposals typically emphasis the former, controlling routine cruise operations costs at the expense of science operations at the target. Then, as planning for the science phase ramps up during cruise, a better understanding of the need for trained, expert personnel for science operations causes cost growth in order to bring in additional staff, or to the accelerate the planned staffing increase to ensure that sufficient staff, and sufficient training opportunities, are available to bring the risk to science operations down to an acceptable level.

\section{Causes and Mitigation}

The operations cost growth drivers discussed above can be divided into management issues and technical issues. The management issues involve the ability to estimate operations development and support resources and management decisions regarding the scheduling of development in support of operations. The technical issues revolve around fully understanding the operation of the spacecraft components in the planned mission environment. In so far as the technical issues generally result from overly optimistic assumptions regarding operational use of heritage and new technologies or inadequate understanding of the mission complexity, coupled with insufficient analysis to confirm these assumptions, these could also be generally considered as management issues involving the allocation of resources and emphasis on mission operations.

In other words, the primary cost growth drivers are not typically technical issues. The "unknown unknowns" are not drivers for the operations cost growth seen in D\&NF missions. For the missions studied, the primary operations cost growth drivers are factors that could have been analyzed, understood, and estimated based on the original or replan operations concept. Thus, the underlying causes for the operations cost growth drivers are managerial in nature, not technical, and any solution or mitigation needs to address management processes. In short, project management needs to ensure that sufficient emphasis is given to operations early enough in the project life cycle to understand, analyze, and realistically cost operational requirements and resources.

While the study was not specifically designed to identify possible causes for the observed operations cost growth drivers, there are a few likely candidates. As a series of independent, unique missions unified only by a goal to further our understanding of the solar system, each new mission is to some extent unique. Thus, there is only a 
limited opportunity, primarily restricted to cruise operations, to build up the wealth of experience, the knowledge base, and the suite of operational tools that make mission operations routine and easily estimated.

The NASA mission selection process is heavily driven by the emphasis on accomplishing the most science for the least cost and risk. Phase E costs are typically small compared to the spacecraft development in phases C and D. Thus, while more emphasis is being placed on overall mission cost risk, traditional project management and selection processes have concentrated on the technical risks during spacecraft development. In addition, there is a perception that cost increases late in the development life cycle (near the start of or during operations) are "safe," that given the money already spent on spacecraft development, NASA will not cancel a project merely because of cost increases during phase E. Finally, proposal and concept study funding is limited; proposal and project managers must carefully manage the available resources and make difficult decisions to ensure that all critical trades and analyses are accomplished. Taken together, these make the operations phase an obvious candidate for project management to accept a greater risk of cost growth by taking an optimistic approach to operations cost estimates or limiting resources for early operations cost trades and analysis.

To reduce the programmatic risk resulting from operations cost growth, project managers need to ensure that sufficient emphasis is placed on estimating operations support development and operations support staffing early in the project life cycle. Project managers and system engineers should be aware of the potential areas of operations cost growth: heritage and new technology assumptions, operational complexity, and science support tools, to ensure that the necessary resources are available to analyze any operational impact or operational support requirement along with equivalent spacecraft analysis in each phase. Finally, project managers need to be aware that operations cost growth is not independent from growth in the overall mission cost. Figure $\mathbf{4}$ compares the percent increase in operations (phase E) costs to the percent increase in total mission life cycle cost. The two are clearly commensurate. While there is no direct correlation between spacecraft development cost growth and operations cost growth (i.e., an increase is spacecraft development cost was not linked to a resulting increase in operations cost), in each case, operations costs stayed proportional to overall LCC even when the overall LCC increased by as much as $50 \%$. Project managers need to be aware of both the potential impact of spacecraft changes on operations, the possibility of secondary impacts (e.

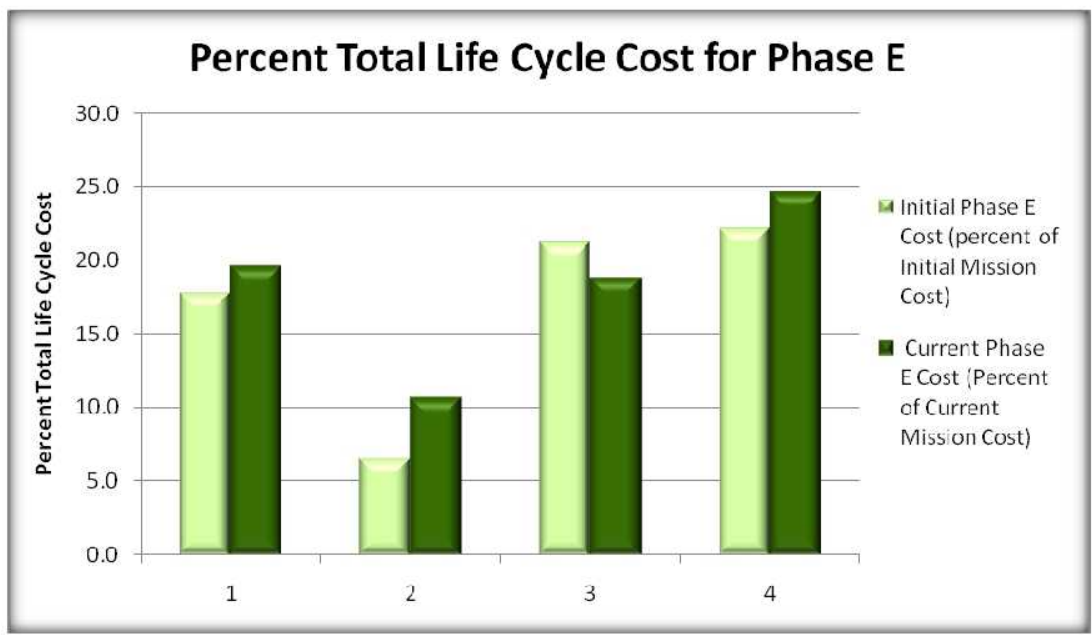

Figure 4. Operations Cost Growth Compared to Total LCC g., increased operational complexity) that can result from mission replans, as well as the potential for growth in phase $\mathrm{E}$ costs independent from any other project issues.

To increase emphasis on early work to develop realistic operations support requirements and cost estimates, the latest Discovery $\mathrm{AO}^{3}$ has been modified to emphasize operations costs and cost risks. Reserves proposed as part of the total LLC have been clearly identified as covering all phases of the mission life cycle, including phase E. Proposer will need to justify the adequacy of the cost reserves and demonstrate an approach for maintaining adequate unencumbered reserves through development and operations. Evaluation of the adequacy and robustness of the technical plan will include the plan for mission operations and the flexibility to recover from problems during both development and operations.

\section{Conclusion}

In spite of increased emphasis over the past 20 years on operability and parallel support for development of operations capabilities and the spacecraft, D\&NF projects are still experiencing significant cost overruns during operations. These overruns are the direct result of late development of operations requirements, overly optimistic assumptions regarding the savings resulting from the use of heritage technology, inadequate analysis of the effect of new technology on operations, delayed completion of ground system development work, and inadequate planning for sustaining engineering and the special requirements of long duration missions (e.g., knowledge retention and 
hardware/software refresh). However, there was no indication in the missions studied that the problem is technical in nature, that new technology is required to solve the operational issues and contain operations cost growth. Rather the study indicates that increased project management attention to operations requirements and staffing estimates from the start of the project life cycle should mitigate to a large extent the cost growth seen in the missions studied. Starting from the earliest initiation of mission formulation, attention to operations concepts and requirements for all aspects of the mission, a realistic look at tool and staffing requirements, and detailed supporting analyses should enable projects to more accurately predict operations costs, and to ensure that those estimates evolve in concert with the overall spacecraft design and development.

\section{Acknowledgments}

The Discovery \& New Frontiers Program Office Life Cycle Cost Study was performed under the direction of Paul Gilbert (MSFC), led by Bryan Barley (MSFC), and supported by Kenny Mitchell (MSFC-retired) and Marilyn Newhouse (CSC).

\section{References}

${ }^{1}$ Barley, B., Gilbert, P., and Newhouse, M., "Improving the Life Cycle Cost Management of Planetary Missions," February 2010.

${ }^{2}$ Fesq, L., Cancro, G. Jones, C., Ingham, M., Leitner, J., McDougal, J., Newhouse, M., Rice, E., Watson, D., Wertz, J., "Spacecraft Fault Management Workshop Results for the Science Mission Directorate, Planetary Sciences Division," March 2009

${ }^{3}$ Draft Discovery 2010 Announcement of Opportunity, December 7, 2010. 


\section{Planning and Estimation of Operations}

\section{Support Requirements}
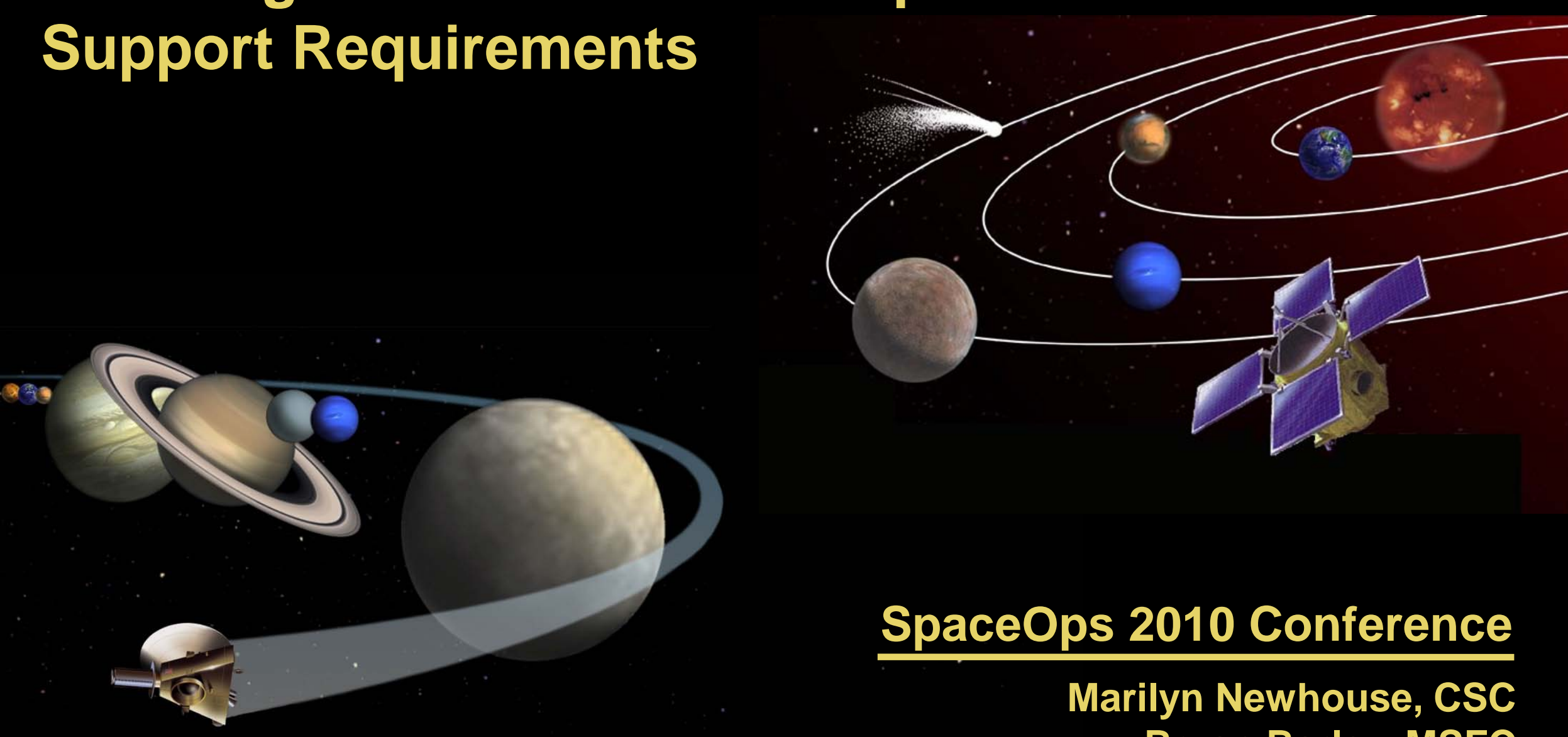

SpaceOps 2010 Conference

Marilyn Newhouse, CSC Bryan Barley, MSFC Allen Bacskay, MSFC Dennon Clardy, MSFC 


\section{D\&NF Program Science Missions}

- The Moon (Lunar Prospector, M³, GRAIL)

- Mars (Mars Pathfinder, ASPERA-3)

- Inner Planets (MESSENGER, Strofio)

- Outer Planets (New Horizons, Juno)

- Comets (CONTOUR, Stardust, Deep Impact, EPOXI, NEXT)

- Asteroids (NEAR, Dawn)

- Interplanetary Space (Genesis)

- Extra-Solar System (Kepler)
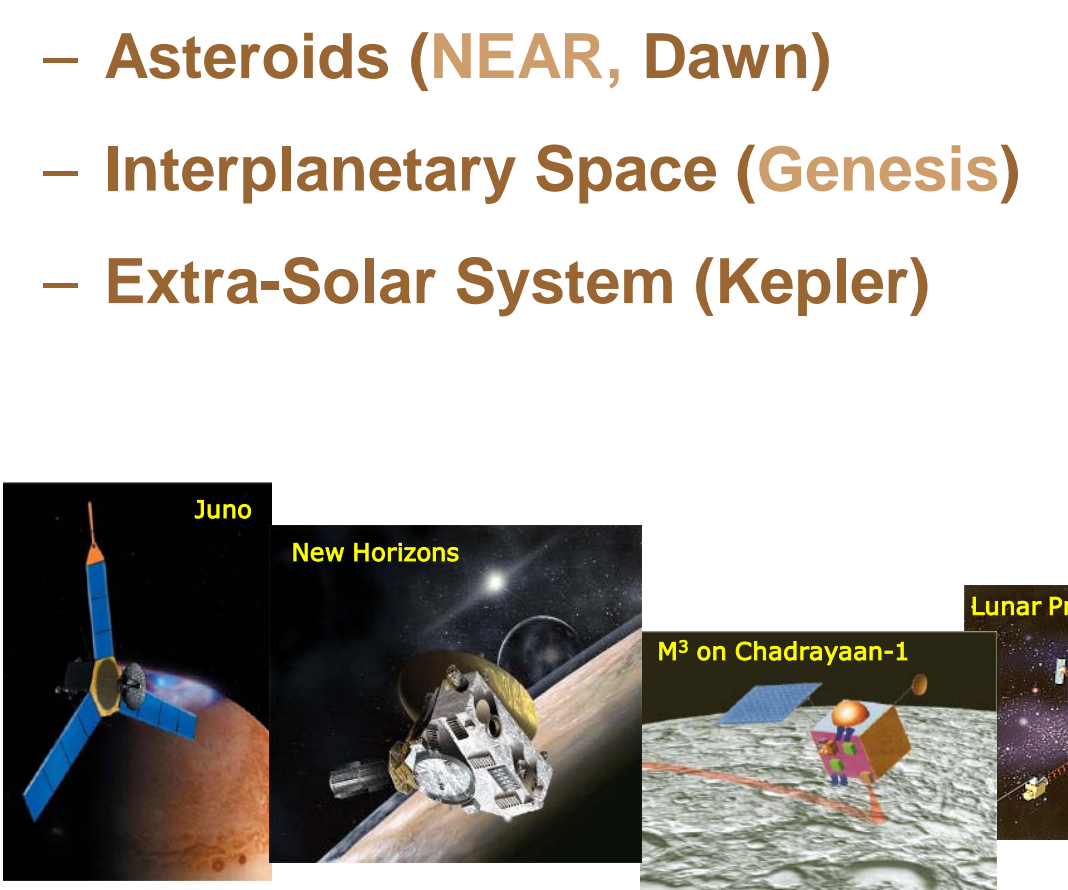


\section{Operations Cost Growth}

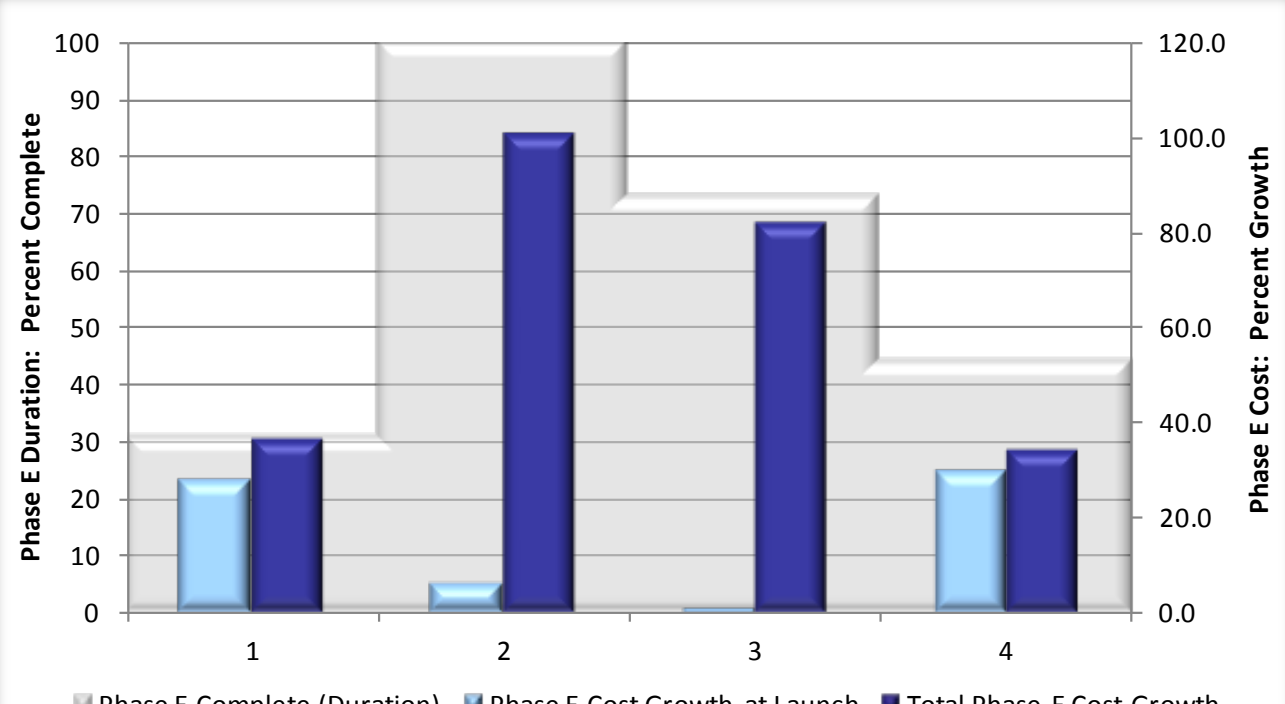

- Four of the five showed growth ranging from $35 \%$ to 100

- Remaining mission controlled cost growth by cutting phase E duration and science support tool development

- Phase E cost growth was commensurate with overall mission life cycle cost (LCC) growth

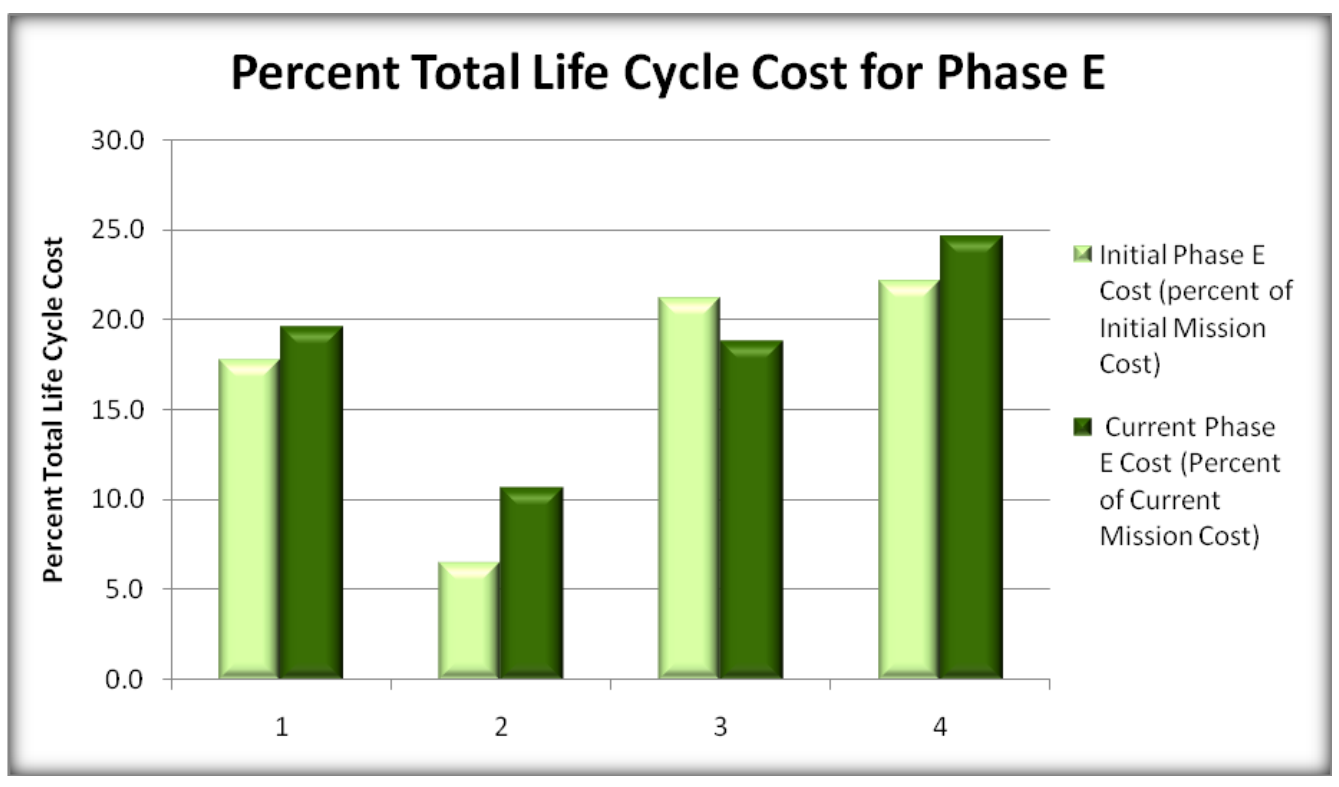




\section{Operations Cost Estimation}

In the missions studied, all five operational missions experienced phase $E$ cost growth resulting from an underestimate of the operational requirements

- Underestimation of the tools required for science operations at the science targets

- Inadequate analysis of and planning for fault protection and fault management capabilities

- Underestimation of the overall complexity of operations activities and therefore the level of support for planning and executing onboard activities

- Overly optimistic assumptions regarding the use of heritage technology

- Over-reliance on heritage/multi-mission operations center systems and capabilities 


\section{Scheduling}

Deep space missions plan to take advantage of the long cruise phase to complete ground system capabilities, particularly science phase capabilities

- Helps maintain critical knowledge and skills during the cruise to the science target

- Increases temptation to underemphasize the science phase requirements and design during project formulation

- Increases the risk that the tools and development effort will be significantly underscoped in early cost estimates

- Allows a decrease in development discipline

- Phase E development rarely implement s earned value management

- Equivalent of CDR -level reviews, including independent experts, are rarely held during operations 


\section{Long Term Operations}

Project proposals do not adequately address requirements for hardware and software refresh over the life of a deep space mission

- Eliminating or minimizing hardware and operating system upgrades can appear to reduce risk

- Assumptions become untenable as a result of operating system upgrades and hardware obsolescence

- Assumptions can actually increase the risk of loss of science in the event that aging hardware fails during a critical mission event

Project proposals typically emphasis controlling routine cruise operations costs

- Detailed science planning during cruise identifies the need for trained, expert personnel for science operations

- Operations costs increase in order to bring in additional staff, or to the accelerate a planned staffing increase 


\section{Operations Cost Growth Drivers}

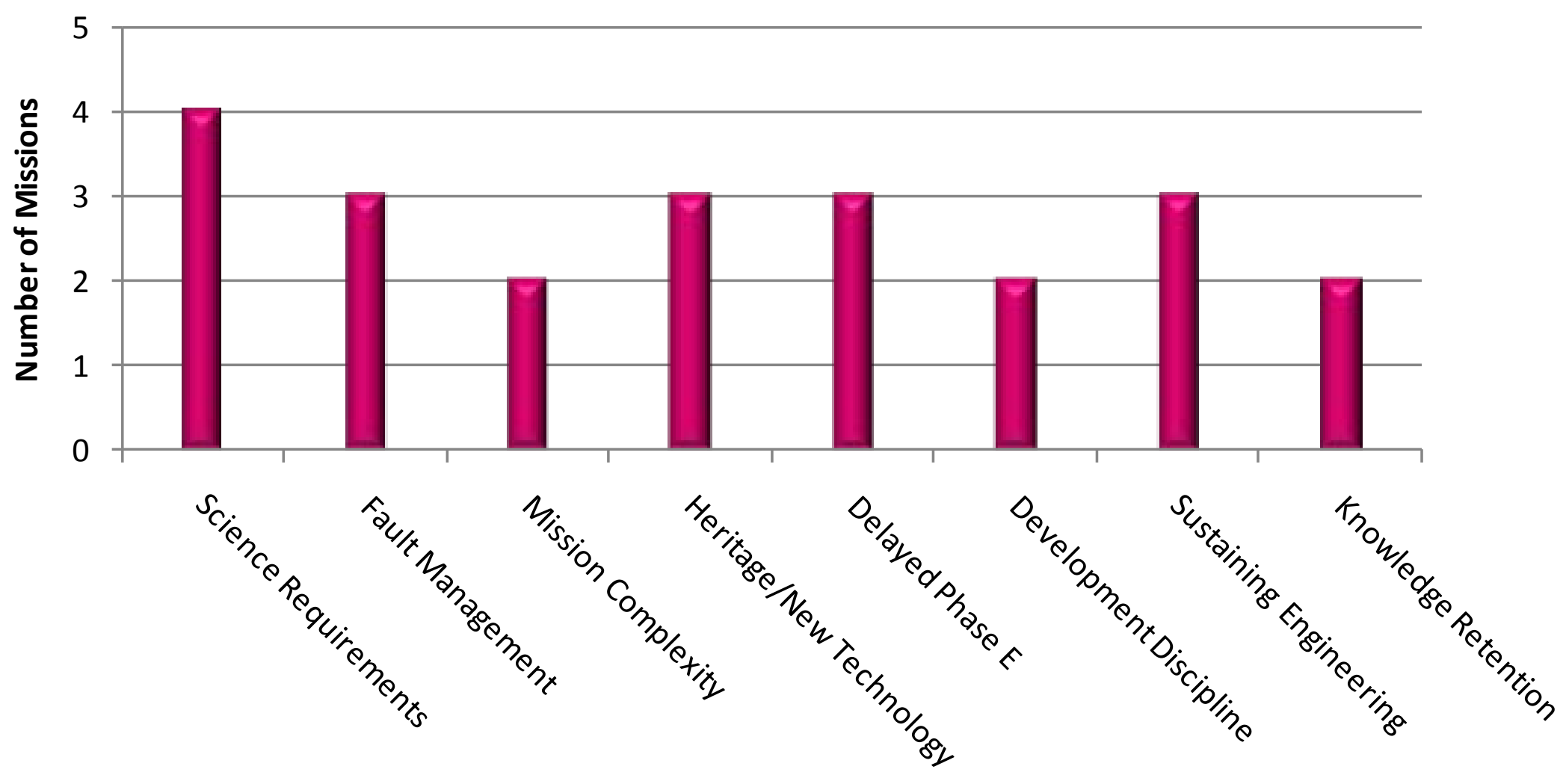




\section{Potential Causes and Mitigations}

The primary cost growth drivers are not typically technical issues; the primary operations cost growth drivers are factors that could have been analyzed, understood, and estimated

\section{Potential causes}

- Each new mission is to some extent unique

- Project management and selection processes have concentrated on the technical risks during spacecraft development

- Perception that cost increases late in the development life cycle (near the start of or during operations) are "safe"

- Proposal and concept study funding is limited

Project management mitigations

- Ensure that sufficient emphasis is placed on estimating operations support development and operations support staffing early in the project life cycle

- Maintain awareness of the potential areas of operations cost growth: heritage and new technology assumptions, operational complexity, and science support tools

- Ensure that the necessary resources are available to analyze the operational characteristics

- Remember that operations cost growth is not independent from growth in the overall mission cost 


\section{Conclusion}

Operations costs are small $(-10-25 \%)$ compared to overall mission life cycle cost, yet overruns decrease a program's ability to sustain future mission flight rates

The majority of the cost growth drivers seen in the D\&NF study could have been avoided or significantly decreased by project management emphasis on early planning and realistic estimation

- Realistic assessment of multi-mission capabilities and additional support required by unique mission characteristics

- Realistic assessment of new and heritage spacecraft technology on operations

- Realistic assessment of overall mission complexity, including fault management, and the ability to reduce operations staff through previous mission experience

- Upfront planning for retaining or re-developing trained staff across a long cruise to the science target

- Carefully trading sustaining engineering and hardware/software refresh across the full mission operations phase against reduced phase E costs

The Discovery and New Frontiers Program Office Life Cycle Cost Study was performed under the direction of Paul Gilbert (MSFC), led by Bryan Barley (MSFC), and supported by Kenny Mitchell (MSFC-retired) and Marilyn Newhouse (CSC) 